\section{Schlechte Urtikariaversorgung in Deutschland}

\author{
Es ist schlimmer als gedacht - das ist die erste Zwischenbilanz der \\ internationalen Real-life-Studie AWARE mit den Daten von über 1.500 \\ deutschen Patienten mit einer H1-Antihistaminika-refraktären chronischen \\ spontanen Urtikaria.
}

Übark ber Krankheitsverlauf, Versorgung und Lebensqualität von Patienten mit einer H1-Antihistaminika-refraktären chronischen spontanen Urtikaria (CSU) ist bisher wenig bekannt. Vor allem die nur leicht oder mäßig betroffenen Patienten werden oft ausschließlich von niedergelassenen Kollegen betreut. Es existiert zwar eine S3-Leitlinie zur Urtikariatherapie, diese geht aber offensichtlich an den Bedürfnissen der Praxis vorbei - viele der leicht bis mäßig betroffenen Patienten gelten als zeitaufwendig und schwierig zu behandeln. Um mehr über dieses bisher in der Forschung eher vernachlässigte Klientel zu erfahren, wurde die derzeit weltweit noch laufende prospektive Nichtinterventionsstudie AWARE („A World-wide Antihistamine-Refractory chronic urticaria patient
Evaluation") initiiert. Nun liegen die ersten Basisdaten von 1.500 AWARE-Teilnehmern aus Deutschland vor.

Einbezogen in die deutsche Kohorte waren erwachsene Urtikariapatienten mit einer H1-Antihistaminika-refraktären CSU, die 2014 in insgesamt 256 Urtikariazentren oder dermatologischen Praxen evaluiert worden waren. $70 \%$ der 1.539 auswertbaren Teilnehmer waren weiblich, das Durchschnittsalter lag bei 46,3 Jahren, 73,9\% der Patienten wurden in niedergelassenen Praxen betreut. Im "Urticaria control test" (UCT), einem der vier eingesetzten patientenberichteten Outcomes (PROs), ergab sich ein Durchschnittswert von 7,9 , rund $77,5 \%$ der Patienten hatten UCT-Werte von unter 12, was auf eine unkontrollierte Erkrankung hinweist. Einer von zwei Patienten hatte ein Angioödem, die häufigsten Komorbiditäten waren eine chronische induzierbare Urtikaria (CindU, 24\%), allergische Rhinitis (18,2\%), Hypertonie (18,1\%), Asthma (12\%) und Depression (9,5\%). $57,6 \%$ der Patienten erhielten mindestens ein Medikament - unter anderem H1Blocker der zweiten $(46,3 \%)$ oder der ersten Generation $(9,1 \%)$ und Glukokortikoide $(15,8 \%)$. Die krankheitsbedingte Lebensqualität war insgesamt schlecht. Die Patienten beanspruchten überdurchschnittlich häufig ärztliche Hilfe in Form von Notfallambulanzen $(29,7 \%)$, Allgemeinärzten $(71,9 \%)$ und zusätzlich Allergologen oder Dermatologen (50,7\%).

Fazit: CSU-Patienten in Deutschland haben oftmals eine schlecht kontrollierte Erkrankung, begleitet von Angioödem, CindU, allergischer Rhinitis und Asthma. Die Lebensqualität ist schlecht, der Verbrauch an ärztlichen Hilfeleistungen hoch.

Dr. Barbara Kreutzkamp

Maurer $\mathrm{M}$ et al. $\mathrm{H} 1$-antihistamine-refractory chronic spontaneous urticaria: it's worse than we thought - first results of the multicenter real-life AWARE study. Clin Exp Allergy 2017; 47: 684-92

\section{Erdnuss-OIT: Rasch eskalieren unter Omalizumab}

Der IgE-Antikörper Omalizumab erlaubt eine rasche Dosissteigerung im Rahmen der spezifischen oralen Nahrungsmittelimmuntherapie. In einer randomisierten placebokontrollierten Doppelblindstudie wurde das speziell bei Erdnussallergikern dokumentiert.

$D^{i}$ e orale Immuntherapie (OIT) ist eine neue Therapieoption für Patienten mit Nahrungsmittelallergie. Allerdings entwickeln vor allem Hochrisikopatienten während der Desensibilisierung häufiger adrenalinpflichtige Reaktionen oder erweisen sich als therapierefraktär. Auch die Compliance leidet bei der langsamen Allergenaufdosierung. Diese Situation lässt sich durch den gleichzeitigen Einsatz des Anti-IgE-Antikörpers Omalizumab verbessern, so die Ergebnisse von drei offen durchgeführten Studien. Nun wurden Wirksamkeit und Sicherheit einer Omalizumab-unterstützten OIT erstmals in einer randomisierten, doppelblinden, placebokontrollierten Studie speziell bei Erdnussallergikern untersucht.
Einbezogen in die Phase-II-Studie waren 39 Patienten, die auf weniger als $50 \mathrm{mg}$ Erdnussprotein eine deutliche Reaktion zeigten. Zwölf Wochen vor Beginn der OIT erhielten sie entweder Omalizumab $(n=29)$ oder Placebo $(n=8)$. Im Rahmen der OIT wurde die Allergendosis in Ein-Wochen-Schritten auf bis zu $2.000 \mathrm{mg} /$ Woche eskaliert, in Woche 20 der IgE-Antikörper abgesetzt und die Allergendosis zunächst beibehalten. Nach weiteren zwölf Wochen ohne Omalizumab-Schutz folgte eine 4.000-mg-Provokation. Wurde auch diese Dosis vertragen, nahmen die Patienten anschließend täglich $4.000 \mathrm{mg}$ Erdnussprotein zu sich.

Schon während der initialen Aufdosierung innerhalb von einem Tag auf eine Allergendosis von $250 \mathrm{mg}$ ergaben sich Vorteile für die OmalizumabGruppe: 23 der 29 Patienten (79\%) unter Verum und nur ein Patient unter Placebo (12\%) erreichten die Allergenzieldosis. Bei diesen Patienten wurde die OIT planmäßig fortgesetzt. 21 Patienten der Verumgruppe und ein Patient der Placebogruppe erreichten dann das primäre Studienziel: Sie vertrugen $2.000 \mathrm{mg}$ Erdnussprotein acht Wochen nach Beendigung der Studienmedikation $(\mathrm{p}<0,01) .23$ Studienteilnehmer unter Verum und ein Teilnehmer unter Placebo tolerierten später auch die 4.000-mg-Provokation.

Fazit: Im Rahmen einer Erdnuss-OIT erlaubt die initial begleitende Applikation des IgE-Antikörpers Omalizumab eine rasche Dosiseskalation.

\section{Dr. Barbara Kreutzkamp}

MacGinnitie AJ et al. Omalizumab facilitates rapid oral desensitization for peanut allergy. J Allergy Clin Immunol 2017; 139: 873-81 